\title{
Трехпараметрическая модель межмолекулярных взаимодействий как основа для классификации и выбора неподвижных фраз для газовой хроматографии
}

\author{
Зайцева Е.А., Долгоносов А.М. \\ Институт геохимии и аналитической химии им. В.И. Вернадского Российской академии наук \\ (ГЕОХИ РАН), Москва
}

Поступила в редакцию 25.09.2019 г.

DOI 10.17308/sorpchrom.2019.19/1167

Описан метод трехпараметрической характеристики селективности газохроматографических неподвижных фаз, который базируется на представлении энергии межмолекулярного взаимодействия вкладами неполярных, полярных сил и водородных связей. Все параметры, используемые в математических выражениях, имеют физический смысл, переносимы и не требуют проведения дополнительных экспериментов. Характеристики неподвижных фаз могут быть рассчитаны как из экспериментальных данных по хроматографическому удерживанию, так и a priori, по известной структурной формуле фазы. Описаны оба способа и проведен расчет параметров для ряда веществ. Полученные разными способами характеристики селективности неподвижных фаз согласуются между собой. Рассматривается также основанная на трехпараметрической модели концепция выбора неподвижной фазы для разделения близких веществ.

Ключевые слова: газовая хроматография, межмолекулярные взаимодействия, неподвижная фаза, полярность, гидрофильность, энергия адсорбции.

\section{Three-parameter model of intermolecular interactions as a basis for classification and selection of gas-chromatographic stationary phases}

\author{
Zaitceva E.A., Dolgonosov A.M. \\ Vernadsky Institute of Geochemistry and Analytical Chemistry Russian Academy of Sciences \\ (GEOKHI RAS), Moscow
}

\begin{abstract}
The present article proposes a mathematical model based on the representation of the intermolecular interaction energy as the sum of three terms: non-polar, polar and hydrogen bond energies, which depend on the parameters of each of the interacting objects - the generalized charge, dipole moment and the probability of hydrogen bond formation. The work is a continuation of the method developed by the authors for describing gas-chromatography stationary phases. The proposed method of stationary phases classification (ThreeParameter Characterization method) differs from existing, mainly theoretical justification, the lack of adjustable parameters and good predictive power; this method does not require special experiments or complex computer calculations, it is very economical to use experimental data.

Selectivity of stationary phase is described by two relative characteristics - polarity and hydrophilic; polarity is the ratio of the square of the dipole moment of the stationary phase to its generalized charge and hydrophilicity, in turn, the probability of formation of a hydrogen bond. Quantitative concepts of polarity and
\end{abstract}


hydrophilicity are introduced, they can be found from experimental data on chromatographic retention indices. Also, these characteristics for objects of intermolecular interaction can be calculated a priori from the molecular structure. The characteristics found are to be plotted on so-called selectivity map with coordinates of polarity and hydrophilicity. Developed method agrees well with the corresponding characteristics of the Rorschneider - McReynolds method. The selectivity map allows choosing the most suitable stationary phase for separation of the target analytes according to the principle «similia similibus solvuntur». This method has a good predictive ability and can be used to predict the behavior of analytes in gas chromatography.

Keywords: intermolecular interaction energy; hydrogen bond; gas chromatography; methods for characterizing the selectivity of stationary phases; polarity; hydrophilicity.

\section{Введение}

В настоящее время в газовой хроматографии (ГХ) применяется большое количество жидких неподвижных фаз (НФ). При выборе НФ имеют значение такие ее свойства, как полярность, способность химически взаимодействовать с исследуемыми веществами, вступать в специфические взаимодействия. Нет необходимости в неограниченном разнообразии НФ, достаточно иметь несколько широко используемых НФ, каждая из которых способна к участию в определенных видах межмолекулярных взаимодействий, и ряд фаз для решения специальных задач, например разделения стереоизомеров. При выборе НФ часто руководствуются известным правилом «подобное растворяется в подобном». Качественный учет возможных в системе межмолекулярных взаимодействий часто позволяет осуществлять обоснованный выбор НФ, повысить эффективность разделения и сократить время анализа. Трудность строгого решения этой задачи привела к тому, что в ГХ широко используются различные эмпирические подходы, например шкалы полярности НФ.

Роршнайдер и МакРейнольдс предложили обобщенную классификацию НФ с использованием разности индексов удерживания для стандартных веществ сравнения. Их способ на данный момент является самым распространенным методом характеристики газохроматографических НФ [1-3].

Индекс удерживания Ковача характеризует удерживание вещества в колонке неподвижной фазой при температуре Т и рассчитывается относительно двух налканов с числом углеродных атомов $m$ и $m+1$ по формуле:

$$
I_{i}=100 \cdot\left(m+\ln \frac{\Gamma_{i}}{\Gamma_{m}} / \ln \frac{\Gamma_{m+1}}{\Gamma_{m}}\right),
$$

где $\Gamma$ - константа Генри, $m$ - индекс $\boldsymbol{t}$-алкана, содержащего $m$ атомов углерода, $I_{i}-$ индекс Ковача рассматриваемой молекулы (с индексом $i$ ), для которой выполняется неравенство $\Gamma_{m} \leq \Gamma_{i} \leq \Gamma_{m+1}$.

Индекс Ковача слабо зависит от параметров режима, в частности, от температуры, что позволяет оценивать порядок хроматографического удерживания разных веществ. Численное значение индексов Ковача определяется лишь физикохимическими свойствами анализируемого вещества, природой неподвижной фазы и температурным режимом колонки, индекс удерживания вещества той или иной неподвижной фазой можно поставить в ряд с такими известными константами, как температура кипения (плавления), плотность или показатель преломления.

Исследуемое соединение характеризуется наименьшим индексом Ковача на сквалане, любая другая жидкая фаза обладает некоторой полярностью, причем полярные силы действуют на соединение в большей степени, чем на н-углеводороды, относительно которых определяются индексы Ковача. Соответствующую разность индексов Ковача, представляющую собой меру полярности жидкой фазы относительно сквалана, Роршнайдер обозначил $\Delta I$ :

$$
\Delta I=I_{s p}-I_{s q}
$$


Вклад полярности в индекс удерживания можно описать линейным уравнением, содержащим ряд констант, зависящих либо от свойств НФ (константы $x, y, z, u$, $s)$, либо от свойств образца (константы от $a$ до $e$ ):

$$
\Delta I=a \cdot X+b \cdot Y+c \cdot Z+d \cdot U+e \cdot S
$$

Для представления набора функциональных групп, с которыми могут взаимодействовать полярные силы, были выбраны 5 стандартных соединений: бензол, этанол, метилэтилкетон, нитрометан и пиридин. Если разделить на 100 каждое значение $\Delta I$, получатся константы Роршнайдера $-X, Y, Z, U, S$ соответственно.

Полуэмпирическая модель сольватационных параметров Абрахама [4-7] внешне схожа со схемой Роршнайдера, однако некоторые ее параметры рассчитываются теоретически. Символьное представление модели сольватационных параметров, введенное Абрахамом, выглядит следующим образом:

$$
S P=c+e E+s S+a A+b B+v V(l L)
$$

где $S P$ - любая величина, связанная с распределением вещества; $E, S, A, B$ и $V$ - так называемые дескрипторы аналита, или независимые переменные, присваиваемые каждому соединению и характеризующие его поляризуемость, дипольность, способность участвовать в образовании водородных связей в качестве донора и акцептора протона соответственно; молекулярный размер соединения учитывается посредством дескрипторов $V$ при изучении распределения между конденсированными фазами и $L$, если одной из фаз является газ; коэффициенты $e, s, a, b$ и $v, l$ - так называемые сольватационные параметры - отражают свойства системы, в которую помещено вещество.

Другие методы имеют всего один оценочный параметр - это различные критерии полярности [8] или схемы гидрофобно-гидрофильного баланса (ГГБ) [9] где НФ имеют всего один оценочный параметр. Например, критерий гидрофобности Шатца [10] или линейно коррелирующий с ним параметр $\log P$ (логарифм коэффициента распределения вещества в системе 1-октанол - вода) используются для оценки баланса гидрофильных и гидрофобных свойств органических соединений. Существует метод априорного расчета $\log P$, запатентованный корпорацией BioByte, который основан на современных квантовохимических представлениях [11].

Отметим недостатки этих методов. Схема Роршнайдера-МакРейнольдса основана, в первую очередь, на предполагаемой независимости пяти видов энергии у пяти эталонных образцов. Однако «чистых» веществ, у которых был бы только один вид взаимодействия, не существует; кроме того, независимых видов межмолекулярной энергии не так много - меньше пяти. Отсюда вытекает ошибочность и избыточность схемы, так как пространство параметров Роршнайдера имеет меньшую размерность, а принимающиеся независимыми характеристики эталонных веществ суммируют вклады разных видов энергии и поэтому не могут служить ортами системы координат. Модель сольватационных параметров за период своего существования зарекомендовала себя в качестве эффективного инструмента исследования, однако, стоит отметить, что модель редко применяется для прогноза времен удерживания при оптимизации хроматографических процессов, так как уравнения просто не обеспечивают необходимой точности. Уравнение учитывает не все возможные специфические взаимодействия, которые встречаются в газовой хроматографии, а между некоторыми параметрами возможны корреляционные зависимости [12-16]. Более того, энергии, ответственные за распределение аналита, крайне малы, что делает их точный прогноз при помощи любой универсальной модели, которой является и модель Абрахама, крайне непростой задачей.

Оба метода не имеют фундаментального обоснования, содержат большое количество подгоночных параметров и требуют трудоемких подготовительных 
экспериментов. Более простые в расчетах критерии полярности и методы ГГБ не отражают в полной мере сложную природу межмолекулярных взаимодействий и поэтому у НФ с разной селективностью эти параметры могут совпадать. В целом, недостатки представленных методов можно свести к следующим: отсутствие теоретического обоснования, ограниченная предсказательная способность методов, экспериментальная трудоемкость. Нельзя забывать еще один важный момент - понятие полярности у каждого из этих методов своё, единого определения полярности НФ нет. Впрочем, ввиду отсутствия альтернативных методов, все эти методы используются при оценке селективности НФ.

Использование теоретических представлений о межмолекулярном взаимодействии для разработки теоретически обоснованного метода характеристики селективности хроматографических фаз является основной темой исследования в работах авторов настоящей статьи [17-19]. Развиваемый подход опирается на фундаментальное описание межмолекулярных взаимодействий и лишен недостатков, присущих традиционным методам: не требуются специальные эталонные адсорбаты, отсутствуют математически некорректные процедуры. Способ описания межмолекулярного взаимодействия в газохроматографической системе использует выражение для общей энергии [20]: $U=U_{n p}+U_{d p}+U_{H}$, где $U_{n p}$ - энергия неполярного взаимодействия это вандерваальсов потенциал типа потенциала Леннард-Джонса, $U_{d p}$ - энергия полярного взаимодействия, которая выражается как сумма ориентационной связи Рейнганума-Кеезома [21-23] и индукционной связи Дебая-Фалькенхагена [24-25], $U_{H}$ - квантовомеханическая оценка энергии Н-связи [18]. Ионные связи не рассматриваются ввиду того, что для газохроматографической системы такие взаимодействия не характерны. В развиваемом методе молекулы характеризуются тремя параметрами: поляризуемостью, пропорциональной обобщенным зарядам $Q$, полярностью, определяемой квадратами дипольных моментов $\mu^{2}$, и вероятностью образования водородных связей $n_{H}$; по числу параметров метод назван методом трехпараметрической характеристики (ТПХ). Такой метод позволяет не только корректно определить характеристики неподвижных фаз по экспериментальным данным об индексах хроматографического удерживания, но и произвести априорный расчет по структурной формуле вещества [26]. Статья посвящена применению теории межмолекулярных взаимодействий для обоснования нового метода классификации НФ и разработки способа выбора НФ, селективной к заданной группе аналитов.

\section{Теоретическая часть}

Используемая в работе теоретическая модель межмолекулярных взаимодействий (MМВ) и, как следствие, модель удерживания молекул на неподвижной фазе (НФ) в газо-жидкостной хроматографии (ГЖХ) базируется на четырех подходах: теории обобщенных зарядов Долгоносова (ТО3) [27,28], описании индукционных взаимодействий Дебая-Фалькенхагена, ориентационных взаимодействий Рейнгаума-Кеезома и квантово-механической оценке снизу абсолютной величины энергии водородной связи. Теория обобщенных зарядов выводит выражение для ван-дерваальсового взаимодействия неполярных молекул типа потенциала Леннард-Джонса, в котором среди теоретических коэффициентов присутствуют характеристики электронного строения молекул - обобщенные заряды. Обобщенные заряды напоминают известные в полуэмпирических подходах атом-атомные потенциалы, однако подчиняются более сложному теоретически обоснованному правилу сумм. В рамках ТО3 не только выводится выражение для энергии ван-дер-ваальсового взаимодействия, 
но и определяется связь обобщенного заряда с поляризуемостью, молекулярной площадкой и т.п.

Квантово-механическая оценка энергии водородной связи базируется на том факте, что Н-связывание сопровождается ростом электронной плотности, а для удержания электрона, создающего эту плотность, необходима потенциальная энергия, превышающая по модулю его кинетическую энергию, которая в силу квантовой неопределенности зависит от размера области распределения электрона. Таким образом, в принятой здесь модели энергия Н-связи оценивается как величина, обратнопропорциональная квадрату ее длины, измеряемой между связываемыми электроотрицательными атомами. В таблице 1 приведены: общее выражение для энергии $\mathrm{MMB}$, формулы вычисления обобщенных зарядов по структуре молекул, поляризуемости, вкладов в энергию неполярных, индукционных и ориентационных сил и водородной связи.

Таблица 1. Теоретические выражения для межмолекулярных взаимодействий

\begin{tabular}{|c|c|c|}
\hline Величина & \multicolumn{2}{|l|}{ Выражение } \\
\hline $\begin{array}{l}\text { Энергия взаимодействия } \\
\text { молекул } 1 \text { и } 2\end{array}$ & $U_{12}=U_{n p 12}+U_{i n 12}+U_{i n 21}+U_{o r 12}+U_{H 12}$ & (1) \\
\hline $\begin{array}{c}\text { Выражение ТОЗ для энер- } \\
\text { гии неполярного взаимо- } \\
\text { действия }\end{array}$ & $\begin{array}{c}U_{n p 12}(r)=Q_{1} Q_{2} u_{b}(r), \\
u_{b}(r)=\frac{e^{2}}{a_{0}}\left[1.938 \cdot 10^{5}\left(r / a_{0}\right)^{-12}-5.115\left(r / a_{0}\right)^{-6}\right]\end{array}$ & (2) \\
\hline $\begin{array}{c}\text { Правило сложения } \\
\text { обобщенных зарядов час- } \\
\text { тей жесткого фрагмента } \\
\text { молекулы } \\
\end{array}$ & $Q^{4 / 3}=\sum Q_{i}^{4 / 3}$ & (3) \\
\hline $\begin{array}{c}\text { Обобщенный заряд жест- } \\
\text { кого фрагмента } i \text { с учетом } \\
\text { мезомерии }\end{array}$ & $Q_{i}=\left(N_{\sigma i}+N_{\pi i} \sqrt{2}-N_{d i} \sqrt{2}\right)^{3 / 4}+\left(N_{d i} \sqrt{2}\right)^{3 / 4}$ & (4) \\
\hline $\begin{array}{c}\text { Обобщенный заряд неже- } \\
\text { сткой молекулы }\end{array}$ & $Q=\sum_{i} Q_{i}$ & (5) \\
\hline $\begin{array}{c}\text { Ограничение на удален- } \\
\text { ность электронов от облас- } \\
\text { ти ММВ - радиус экрани- } \\
\text { рования }\end{array}$ & $\begin{array}{c}N\left(R \geq r_{s}\right)=0, \\
r_{s}=1.710 \sqrt{a_{0} e / \phi}\end{array}$ & (6) \\
\hline $\begin{array}{c}\text { Средний потенциал ва- } \\
\text { лентных электронов } \\
\text { молекулы }\end{array}$ & $\phi \approx \bar{\phi}=\left(e N_{b}\right)^{-1} \sum_{i=1}^{N_{b}} E_{i} / z_{i}$ & (7) \\
\hline Поляризуемость молекулы & $\alpha=a Q, a=4.948 a_{0}^{3}$ & (8) \\
\hline $\begin{array}{c}\text { Энергия индукционного } \\
\text { взаимодействия } \\
\end{array}$ & $U_{\text {in } 12}+U_{\text {in } 21}=-\left(\alpha_{2} \mu_{1}^{2}+\alpha_{1} \mu_{2}^{2}\right) r^{-6}$ & (9) \\
\hline $\begin{array}{c}\text { Энергия ориентационного } \\
\text { взаимодействия }\end{array}$ & $U_{o r 12}=-\frac{2 \mu_{1}^{2} \mu_{2}^{2}}{3 k_{B} T} r^{-6}$ & $(10)$ \\
\hline $\begin{array}{c}\text { Энергия и вероятность } \\
\text { водородной связи между } \\
\text { молекулой и НФ }\end{array}$ & $U_{H 12} \approx-\frac{3 e^{2} a_{0}}{16} \frac{n_{H 12}}{r^{2}}, n_{H 12} \equiv n_{H m} n_{H s p}$ & (11) \\
\hline $\begin{array}{l}\text { Вероятность Н-связи (ин- } \\
\text { дексы: «1»- аналит, «2»- } \\
\text { фаза; «a», «d»- акцептор, } \\
\text { донор Н-связи) }\end{array}$ & $n_{H 12}=\frac{\sqrt{Q_{a 1} Q_{d 2}}+\sqrt{Q_{d 1} Q_{a 2}}}{2\left(Q_{a 1} Q_{d 1}\right)^{1 / 4} \sqrt{Q_{m o l 2}}}$ & (12) \\
\hline
\end{tabular}

Обозначения: $a_{0}$ - радиус Бора, $e$ - элементарный заряд (система СГСЭ), $r$ - расстояние между молекулами, $N_{\sigma i}, N_{\pi i}$ - числа электронов $\sigma$ - и $\pi$-связей, удаленных от места взаимодействия не 
дальше радиуса экранирования $r_{s}, N_{d i}$ - половина числа мезомерных электронов; $\phi$ - потенциал валентного электрона, определяемый энергией ковалентной связи $E_{i}$, в которой участвует $z_{i}$ электронов $\left(z_{i}\right.$ - удвоенная кратность связи); $N_{b}$ - число ковалентных связей в молекуле; $\mu$-модуль дипольного момента молекулы; $n_{H m}$ и $n_{H s p}$ - факторы участия в Н-связи молекулы и НФ, соответственно.

Расчет обобщенного заряда молекулы с помощью формул (3-5) содержит три момента: 1. определение нежестких связей, по которым происходит внутримолекулярное вращение, и разбиение молекулы на жесткие фрагменты (часто оказывается, что нежесткие связи отсутствуют и вся молекула жесткая); 2. выявление мезомерии и числа делокализованных $\pi$-электронов; 3 . определение горизонта экранирования электронов молекулы по формулам $(6,7)$.

Из сравнения выражений для дисперсионной энергии в ТОЗ и в теории Лондона следует (8) - поляризуемость пропорциональна обобщенному заряду [27].

Показатель водородной связи $n_{H 12}$ между молекулой аналита и жидкой фазой имеет вид (12), где $Q_{m o l}$ - О3 молекулы или мономера жидкой фазы, $Q_{a}$ и $Q_{d}$ обобщенные заряды акцепторных и донорных (гидридных) атомов молекулы (или мономера), соответственно. В простом случае аналита только с донорными атомами, которые одновременно являются и акцепторными (спирты, амины, меркаптаны и т.п.), формула (12) упрощается: $n_{H 12}=\frac{\sqrt{Q_{d 2}}+\sqrt{Q_{a 2}}}{2 \sqrt{Q_{\text {mol }}}}$. В этом случае фактор участия в Н-связи для молекулы аналита равен 1 , а $n_{H 12}$ является характеристикой только неподвижной фазы $\left(n_{H s p}=n_{H 12}\right)$.

Если НФ является одним из объектов $\mathrm{MMB}$, в выражения таблицы 1 подставляются характеристики: $Q_{s p}, \mu_{s p}^{2}, n_{H s p}$. В результате получим выражения для энергии сорбции в ГЖХ, приведенные в таблице 2.

Таблица 2. Теоретические выражения для энергии сорбции

\begin{tabular}{|c|c|c|}
\hline 1 & \multicolumn{2}{|c|}{ Выражение } \\
\hline Величина & 2 & \\
\hline $\begin{array}{c}\text { Сорбция как межмо- } \\
\text { лекулярное взаимо- } \\
\text { действие }\end{array}$ & $U_{m, s p}=U_{n p(m, s p)}+U_{i n(m, s p)}+U_{i n(s p, m)}+U_{o r(m, s p)}+U_{H(m, s p)}$ & $(13)$ \\
\hline $\begin{array}{c}\text { Параметры молекулы } \\
\text { Параметры непод- } \\
\text { вижной фазы }\end{array}$ & $Q_{m}, \mu_{m}, n_{H m}$ & \\
\hline $\begin{array}{c}\text { Энергия взаимодей- } \\
\text { ствия молекулы и НФ }\end{array}$ & $Q_{s p}, \mu_{s p}, n_{H s p}$ & \\
\hline $\begin{array}{c}\text { Условие минимума } \\
\text { энергии при равнове- } \\
\text { сии }\end{array}$ & $U_{m, s p}(r)=F\left(Q_{m}, \mu_{m}, n_{H m} ; Q_{s p}, \mu_{s p}, n_{H s p} ; r\right)$ & (14) \\
\hline $\begin{array}{c}\text { Выражение для рав- } \\
\text { новесного расстояния }\end{array}$ & \begin{tabular}{c}
$A x^{10}+B x^{6}-1=0$, \\
\hline$r_{b}, A=21.88 w w_{0}, B=1+c\left(v+v_{0}+c_{T} v_{0} v\right)$
\end{tabular} & (15) \\
\hline Коэффициенты & $x_{0} \approx B^{-1 / 6}$ & (16) \\
\hline
\end{tabular}




\begin{tabular}{|c|c|c|}
\hline 1 & 2 & \\
\hline $\begin{array}{c}\text { Характеристики по- } \\
\text { лярности }\end{array}$ & $v=\frac{\mu_{m}^{2}}{Q_{m}} ; v_{0}=\frac{\mu_{s p}^{2}}{Q_{s p}}$ & (18) \\
\hline $\begin{array}{c}\text { Характеристики гид- } \\
\text { рофильности }\end{array}$ & $w=\frac{n_{H m}}{Q_{m}} ; w_{0}=\frac{n_{H s p}}{Q_{s p}}$ & (19) \\
\hline $\begin{array}{c}\text { Безразмерная энергия } \\
\text { сорбции как отноше- } \\
\text { ние к неполярной } \\
\text { энергии }\end{array}$ & $u\left(x_{0}\right)=6 A x_{0}^{-2}+\left(2 B-x_{0}^{-6}\right) x_{0}^{-6}$ & (20) \\
\hline $\begin{array}{l}\text { Структура безразмер- } \\
\text { ной энергии сорбции }\end{array}$ & $u=1+u_{p}+u_{h}$ & (21) \\
\hline $\begin{array}{l}\text { Вклад полярности в } \\
\text { энергию сорбции }\end{array}$ & $u_{p}=B^{2}-1$ & (22) \\
\hline $\begin{array}{c}\text { Вклад гидрофильно- } \\
\text { сти в энергию сорб- } \\
\text { ции }\end{array}$ & $u_{h} \equiv u-B^{2} \approx 6 A B^{1 / 3}$ & (23) \\
\hline
\end{tabular}

Энергия (14) здесь не приведена в явном виде из-за громоздкости выражения, которое представляет собой сумму членов типа (2,9-11). Условием сорбционного равновесия является равенство нулю градиента энергии (14). В результате решения этого уравнения определены равновесные расстояние (16) и энергия (20) в безразмерном виде. Параметры молекулы и НФ представлены характеристиками полярности (18) и гидрофильности (19). Характеристики полярности и гидрофильности молекул определяются из их структуры путем расчета обобщенных зарядов, дипольных моментов и $n_{H m}$ по формуле $n_{H m}=n_{H 12} / n_{H s p}$. Гидрофобные молекулы с $n_{H m}=0$ отнесены к классу $M_{0}$, а гидрофильные молекулы с $n_{H m} \neq 0$ отнесены к классу $M_{1}$.

Среди применяемых НФ доноры Н-связи часто отсутствуют, в этом случае при образовании Н-связи между молекулами аналита и НФ первая является донором, а вторая - акцептором Н-связи. В формулу (12) для аналита подставляются электроотрицательные атомы из гидридных групп, а для НФ - дегидрированные электроотрицательные атомы. Имеется два исключения из этого правила. 1. Хотя НФ обычно не имеет доноров Н-связи, стеклянные и силикагелевые носители содержат гидроксильные группы, что при оголении их поверхности придает НФ дополнительную гидрофильность. В этом случае для расчета чисел $n_{H m}$ и $n_{H s p}$ надо брать как доноры, так и акцепторы Н-связи. 2. В определенных условиях ГЖХ, например, при температурах не сильно превышающих $100^{\circ} \mathrm{C}$, молекулы аналита могут нести гидратную воду. В таких случаях для расчета числа $n_{H m}$ также надо брать как доноры, так и акцепторы Н-связи. Ниже мы не рассматриваем эти исключения.

Без знания структуры прямое определение полярности и гидрофильности НФ невозможно. Однако эти характеристики НФ можно рассчитать из экспериментальных данных по хроматографическому удерживанию молекул указанных двух классов. В таблице 3 даны формулы для определения безразмерной энергии сорбции и характеристик неподвижной фазы из экспериментальных данных по индексам удерживания (Ковача).

Связь энергии сорбции с индексом Ковача (25), следующая из ТО3, более точна, чем обычно рассматриваемая линейная связь, предполагающая аддитивность атом-атомных потенциалов. Однако, как показано в [29], формула (25) ненадежна для циклических молекул, испытывающих топологические ограничения в фазах 
ГЖХ, молекулы которых содержат атомы водорода [30]. По этой причине циклические (включая ароматические) молекулы в развиваемом методе классификации НФ здесь не рассматриваются.

Таблица 3. Формулы для расчета энергии сорбции, ее компонент и характеристик НФ из экспериментальных данных

\begin{tabular}{|c|c|c|}
\hline Величина & Выражение & \\
\hline $\begin{array}{c}\text { Индекс Ковача (по } \\
\text { данным экспери- } \\
\text { мента) }\end{array}$ & $\begin{array}{c}I_{m}=100 \cdot\left(n+\frac{\ln k_{m}-\ln k_{n}}{\ln k_{n+1}-\ln k_{n}}\right) \\
k_{n} \leq k_{m} \leq k_{n+1}-\text { факторы удерживания молекулы } m \text { и } \\
H \text {-алканов с } n \text { и } n+1 \text { атомами углерода }\end{array}$ & (24) \\
\hline $\begin{array}{c}\text { Приближенная } \\
\text { связь энергии } \\
\text { сорбции молекулы } \\
\text { с индексом Ковача } \\
\end{array}$ & $u \approx Q_{m}^{-1}\left(0.06 I_{m}+2\right)^{3 / 4}$ & (25) \\
\hline $\begin{array}{c}\text { Класс неполярных } \\
\text { молекул }\end{array}$ & $\mu^{2}=0, u \equiv 1$ & (26) \\
\hline $\begin{array}{l}\text { Вклад полярности } \\
\text { для класса } M_{0} \text { гид- } \\
\text { рофобных молекул } \\
\quad(w=0)\end{array}$ & $u_{p}=u-1$ & (27) \\
\hline $\begin{array}{c}\text { Полярность НФ по } \\
\text { данным для моле- } \\
\text { кул класса } M_{0}\end{array}$ & $v_{0 i}=\frac{\sqrt{u_{i}}-1-c v_{i}}{c\left(1+c_{T} v_{i}\right)}, \quad v_{0}=\frac{\sum v_{0 i} v_{i}}{\sum v_{i}}$ & (28) \\
\hline $\begin{array}{l}\text { Вклад гидрофиль- } \\
\text { ности в энергию } \\
\text { сорбции } \\
\end{array}$ & $\begin{array}{c}u_{h}=u-B^{2}=u-\left[1+c\left(v+v_{0}+c_{T} v v_{0}\right)\right]^{2} \\
u_{h} \approx 6 A B^{1 / 3}=131.3 w_{0} w\left[1+c\left(v+v_{0}+c_{T} v v_{0}\right)\right]^{1 / 3}\end{array}$ & (29) \\
\hline $\begin{array}{l}\text { Произведение гид- } \\
\text { рофильностей по } \\
\text { данным для моле- } \\
\text { кулы класса } M_{1}\end{array}$ & $\begin{array}{l}S_{i}=\left(\frac{u-B^{2}}{131.3 B^{1 / 3}}\right)_{i}= \\
=7.62 \cdot 10^{-3} \frac{u_{i}-\left[1+c\left(v_{i}+v_{0}+c_{T} v_{i} v_{0}\right)\right]^{2}}{\left[1+c\left(v_{i}+v_{0}+c_{T} v_{i} v_{0}\right)\right]^{1 / 3}}\end{array}$ & (30) \\
\hline $\begin{array}{l}\text { Гидрофильность } \\
\text { НФ как среднее }\end{array}$ & $w_{0}=\frac{\sum S_{i}}{\sum w_{i}}$ & (31) \\
\hline
\end{tabular}

При расчете характеристики полярности НФ по (28) надо брать данные для полярных молекул класса $M_{0}$, исключая молекулы других классов. Отсюда следует, что при усреднении результатов вычислений по (28) для разных молекул весовым множителем должна быть полярность молекул. Расчет гидрофильности НФ по (31) возможен только после вычисления полярности НФ.

Развиваемый метод можно связать с традиционными методами Роршнайдера - МакРейнольдса приблизительными связями через выражения для приращения индекса Ковача стандартных веществ (эталонов), имеющих свойства полярных и гидрофильных молекул. Связи неточные из-за распределения искомых свойств между несколькими эталонами методики МакРейнольдса, в которой инкременты индекса Ковача частично перекрываются (см. формулу (III)). Если пренебречь такой неточностью, то получим следующие связи (таблица 4).

Если пренебречь малосущественными величинами индукционного взаимодействия, то уравнение (14) для энергии сорбции можно представить как скалярное произведение трехмерных векторов с компонентами, пропорциональными парамет- 
рам $Q, \mu^{2}$ и $n_{H}$ каждого из взаимодействующих объектов. Скалярное произведение выражается через длины векторов и угол между направлениями: $(\mathbf{A} \cdot \mathbf{X})=A X \cos \varphi$. Одинаковые длины векторов А соответствуют близким по сорбционным свойствам молекулам. То же относится и к векторам X, характеризующим НФ.

Таблица 4. Приближенные связи между параметрами селективности НФ в новом и традиционном методах.

\begin{tabular}{|c|c|c|}
\hline Величина & Выражение & \\
\hline \multicolumn{3}{|c|}{ Выражения традиционных методов } \\
\hline $\begin{array}{l}\text { Характеристики мо- } \\
\text { лекул эталонов, аналита и } \\
\text { НФ }\end{array}$ & $\begin{array}{c}\mathbf{E t}_{e}=\left\{\delta_{e j}\right\}, \quad \delta_{e j}=\left\{\begin{array}{l}100 ; j=e \\
0 ; j \neq e\end{array},\right. \\
\mathbf{A}=\left\{a_{j}\right\} ; \mathbf{X}=\left\{x_{j}\right\}\end{array}$ & $(31)$ \\
\hline $\begin{array}{c}\text { Приращение индекса } \\
\text { удерживания аналита на } \\
\text { заданной фазе в сравнении } \\
\text { со стандартной НФ }\end{array}$ & $\Delta I=(\mathbf{A} \cdot \mathbf{X})$ & $(32)$ \\
\hline $\begin{array}{c}\text { Инкремент эталона } \\
\text { «е» для характеристики НФ }\end{array}$ & $\Delta I_{e}=(\mathbf{E t} \cdot \mathbf{X})=x_{e}$ & $(33)$ \\
\hline \multicolumn{3}{|c|}{ Приближенная связь между методами классификации фаз } \\
\hline $\begin{array}{c}\text { Связь между метода- } \\
\text { ми расчета через инкремент } \\
\text { эталона «е» }\end{array}$ & $\Delta I_{e}=x_{e}=16.67 Q_{e}^{4 / 3}\left(u_{e}^{4 / 3}-1\right)$ & $(34)$ \\
\hline $\begin{array}{l}\text { Расчет безразмерной } \\
\text { энергии сорбции по инкре- } \\
\text { менту эталона }\end{array}$ & $u_{e} \approx\left(1+0.06 x_{e} Q_{e}^{-4 / 3}\right)^{3 / 4}$ & $(35)$ \\
\hline $\begin{array}{c}\text { Полярность НФ (28) } \\
\text { по данным для молекулы } \\
\text { кетона }(e=3)\end{array}$ & $v_{0}=\frac{\sqrt{u_{3}}-1-c v_{3}}{c\left(1+c_{T} v_{3}\right)}$ & $(36)$ \\
\hline $\begin{array}{l}\text { Гидрофильность НФ } \\
(31) \text { по данным для молеку- } \\
\text { лы спирта }(e=2)\end{array}$ & $w_{0}=7.62 \cdot 10^{-3} \frac{u_{2}-\left[1+c\left(v_{2}+v_{0}+c_{T} v_{2} v_{0}\right)\right]^{2}}{w_{2}\left[1+c\left(v_{2}+v_{0}+c_{T} v_{2} v_{0}\right)\right]^{1 / 3}}$ & $(37)$ \\
\hline
\end{tabular}

Рост энергии сорбции приводит к повышению селективности НФ к молекуле. Рассматривая множество молекул, близких по удерживанию на заданной фазе, мы получим максимальную величину скалярного произведения для той молекулы, у которой угол $\varphi$ с вектором НФ равен нулю. Условие параллельности векторов, характеризующих свойства веществ, служит количественным объяснением известного в химии правила выбора хороших растворителей - «подобное в подобном». Переход к параметрам полярности и гидрофильности эквивалентен проецированию указанных векторов на плоскость карты селективности. В силу сказанного, наилучшими по селективности для заданной молекулы являются фазы, точки которых на карте селективности находятся на луче, проведенном из начала координат к точке с характеристиками молекулы и при этом максимально к ней приближены.

\section{Эксперимент}

1. Расчет характеристик молекул по структурной формуле. Для получения характеристик полярности и гидрофильности молекулы необходимо:

1) Найти дипольный момент молекулы (справочные данные, молекулярное моделирование [31]); 

вия (5);

2) Рассчитать обобщенный заряд молекулы по формулам $(3,4)$, с учетом усло-

3) Рассчитать полярность молекулы по формуле (18);

4) Рассчитать показатель Н-связи $n_{H}$ по формуле (12);

5) Рассчитать гидрофильность по формуле (19).

2. Теоретическая оценка характеристик селективности НФ. Для получения характеристик НФ по ее структурной формуле необходимо:

1) Выбрать фрагменты молекулярной структуры НФ, которые отображают химический состав и усреднить их характеристики. Для полимеров рассматривали структуру мономера.

2) Для выбранного фрагмента рассчитать обобщенный заряд по формуле, выведенной в работе [32]:

$$
Q_{s p}=\frac{\alpha e}{3}\left(a_{0} E_{1}\right)^{-3 / 2},
$$

где $E_{1}=e \phi-$ средняя энергия, приходящаяся на 1 электрон химической связи в фрагменте НФ, определяемая по формуле (7), коэффициент $\alpha=\left(\frac{V_{0}}{V_{0}-s}\right)^{3 / 4}=\frac{\left(Q^{4 / 3}+s\right)^{3 / 4}}{Q}$, $s$ - число экранированных электронов, т.е. число валентных электронов, которые удалены от точки рассматриваемого межмолекулярного взаимодействия на расстояние большее, чем $r_{s}(6)$;

3) Определить дипольный момент выбранного фрагмента НФ. При отсутствии справочных данных, использовали компьютерные программы молекулярного моделирования;

4) Рассчитать полярность НФ $v_{0}$ по (18);

5) Рассчитать показатель Н-связи $n_{H}$ по формуле (12);

6) Рассчитать гидрофильность НФ $w_{0}$ по (19).

3. Расчет характеристик НФ из экспериментальных данных. Расчет характеристик селективности НФ по выражениям таблицы 3 проводился по характеристикам молекул аналитов и их индексам Ковача, экспериментально полученным для исследуемой НФ. Для большей точности расчета использовали индексы Ковача, полученные при одной температуре (здесь: $120^{\circ} \mathrm{C}$ ). Все множество молекул разбивали по классам гидрофильности. Молекулы, у которых отсутствуют атомы-доноры Н-связи относили к классу $M_{0}$, а молекулы, имеющие эти атомы - к классу $M_{1}$. Методика расчета позволяет определить характеристики НФ по данным всего двух полярных молекул - по одной из множеств $M_{0}$ и $M_{1}$. Для этого необходимо:

1) Рассчитать $v_{0}$ по формулам (28) для множества молекул $M_{0}$;

2) Рассчитать гидрофильность фазы по данным для молекул множества $M_{l}$; по формулам $(30,31)$.

В работе все данные по дипольным моментам приводятся в Д, а по полярности - в Д ${ }^{2}$. Для удобства эти размерности ниже опущены. С учетом этого, для коэффициентов (17) брали следующие значения: $\mathrm{c}=0.1498, \mathrm{c}_{\mathrm{T}}=16.78$.

\section{Обсуждение результатов}

Применение разработанного метода ТПХ и построение карты селективности для классификации хроматографических фаз. Классификация НФ по методу ТПХ проводится по характеристикам полярности и гидрофильности, которые отображаются точками на карте селективности (рис. 1). Карта селективности хроматографи- 
ческих фаз строится в координатах $v_{0}-w_{0}$ (см. таб.1; для удобства каждая из величин умножена на 100). Данные для расчета взяты из [33]. На рисунке 1 представлена карта селективности хроматографических фаз, рассчитанная по индексам Ковача для молекул 2-пентанона и 1-бутанола, которые являются двумя из пяти реперных молекул метода Роршнайдера-МакРейнольдса. В методе ТПХ выбранные молекулы представляют множества $M_{0}$ и $M_{1}$, соответственно.

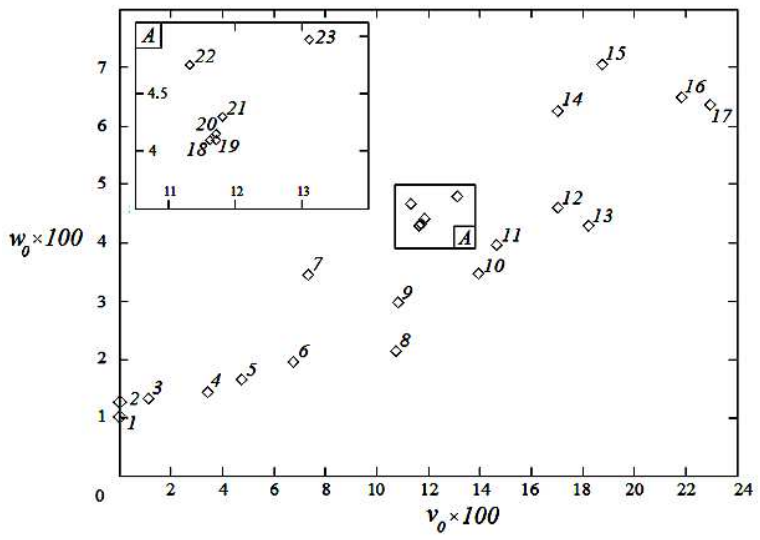

Рис. 1. Карта селективности хроматографических фаз, рассчитанная по индексам Ковача для двух молекул: 1-бутанола и 2-пентанона (см. таблицу 1). Фазы представлены в порядке увеличения полярности: 1 - Squalane, 2 - Apiezon L, 3 - Versilub F-50, 4 - OV-7, 5 - DC-710, 6 - OV-25, 7 - Ucon 50 HB-280X, 8 - OV-225, 9 - XE-60,

10 - Silar-5CP, 11 - XF-1150, 12 - Reoplex 400, 13 - Silar-7CP, 14 - Diglycerol, 15 - THEED, 16 - TCEP, 17 - Cyanoethylsucrose; блок A: 18 - PEG 20M, 19 - PEG 20MTPA, 20 - PEG 6000, 21 - PEG 4000, 22 - Quadrol, 23 - PEG 1000.

Таблица 5. Значения полярности и гидрофильности НФ, рассчитанные по индексам Ковача для молекул 2-пентанона и 1-бутанола.

\begin{tabular}{|c|c|c|c|c|}
\hline № & Название НФ & Состав НФ & $v_{0}$ & $w_{0}$ \\
\hline 1 & 2 & 3 & 4 & 5 \\
\hline 1 & Squalane & $\begin{array}{c}2,6,10,15,19,23- \\
\text { гексаметилтетракозан }\end{array}$ & 0 & 0.010 \\
\hline 2 & Apiezon L,M & 100\% диметилсилоксан & 0 & 0.012 \\
\hline 3 & Versilub F-50 & 100\% диметилсилоксан & 0.011 & 0.014 \\
\hline 4 & OV-7 & $\begin{array}{l}20 \% \text { фенил } 80 \% \text { метил поли- } \\
\text { силоксан }\end{array}$ & 0.034 & 0.015 \\
\hline 5 & DC-710 & $\begin{array}{c}50 \% \text { фенил 50\% } \\
\text { диметилполисилоксан }\end{array}$ & 0.047 & 0.017 \\
\hline 6 & OV-25 & $\begin{array}{c}75 \% \text { фенил } 25 \% \text { метил поли- } \\
\text { силоксан }\end{array}$ & 0.067 & 0.019 \\
\hline 7 & Ucon 50HB-280X & Полиоксиэтиленоксипропилен & 0.079 & 0.032 \\
\hline 8 & OV-225 & $\begin{array}{c}25 \% \text { цианопропил } 25 \% \text { фенил } \\
50 \% \text { метил полисилоксан }\end{array}$ & 0.107 & 0.021 \\
\hline 9 & XE-60 & $\begin{array}{c}25 \% \text { цианоэтил } 75 \% \text { метил } \\
\text { полисилоксан }\end{array}$ & 0.108 & 0.028 \\
\hline 10 & Silar-5 CP & $\begin{array}{c}50 \% \text { цианопропил 50\% фенил } \\
\text { полисилоксан }\end{array}$ & 0.139 & 0.033 \\
\hline 11 & $\mathrm{XF}-1150$ & $\begin{array}{c}50 \% \text { цианоэтил } \\
\text { 50\% метил полисилоксан }\end{array}$ & 0.146 & 0.037 \\
\hline 12 & Reoplex 400 & $\begin{array}{c}\text { Пропиленгликоль-1,2- } \\
\text { адипинат }\end{array}$ & 0.170 & 0.044 \\
\hline
\end{tabular}




\begin{tabular}{|c|c|c|c|c|}
\hline 1 & 2 & 3 & 4 & 5 \\
\hline 13 & SILAR-7 CP & Цианопропилсиликон & 0.182 & 0.042 \\
\hline 14 & Diglycerol & Диглицерол чистый & 0.170 & 0.055 \\
\hline 15 & THEED & $\begin{array}{l}\text { Tetrahydroxyethyl- ethylene } \\
\text { diamine }\end{array}$ & 0.187 & 0.061 \\
\hline 16 & TCEP & $\begin{array}{l}1,2,3 \text {-Tris(2-cyanoethoxy)- } \\
\text { propane }\end{array}$ & 0.218 & 0.057 \\
\hline 17 & Cyanoethylsucrose & Cyanoethylsucrose & 0.229 & 0.056 \\
\hline 18 & PEG 20M & $\begin{array}{c}\text { Полиэтиленгликоль (MW } \\
=20000)\end{array}$ & 0.116 & 0.040 \\
\hline 19 & PEG 20M-TPA & Carbowax 20M terephthalic acid & 0.116 & 0.040 \\
\hline 20 & PEG 6000 & $\begin{array}{c}\text { Полиэтиленгликоль (MW } \\
=6000)\end{array}$ & 0.117 & 0.041 \\
\hline 21 & PEG 4000 & $\begin{array}{c}\text { Полиэтиленгликоль (MW } \\
=4000)\end{array}$ & 0.118 & 0.041 \\
\hline 22 & Quadrol & $\begin{array}{c}\text { N,N,N',N'-Tetrakis(2- } \\
\text { hydroxypropyl)ethylenediamine }\end{array}$ & 0.113 & 0.043 \\
\hline 23 & PEG 1000 & $\begin{array}{c}\text { Полиэтиленгликоль (MW } \\
=1000)\end{array}$ & 0.131 & 0.044 \\
\hline
\end{tabular}

Карту селективности можно разделить условно на 12 областей: 4 - по абсцисce (P1: 0-2 слабо-, Р2: 2-8 средне-, Р3: 8-16 сильно-, Р4: 16-24 высокополярные) и 3 по ординате (Н1: 0-2 слабо-, Н2: 2-5 средне- и Н3: 5-8 сильногидрофильные). К группе Р1Н1 относятся фазы №№ 1, 2 и 3, фазы №№ 4, 5, 6 относятся к группе Р2Н1, фаза № 7 - к группе Р2Н2, фаза № 8 - к группе Р3Н1, фазы №№ 9-11 и 18-23 - к группе Р3Н2, фазы №№ 12,13 - к группе Р4Н2 и фазы №№ 14-17 - к группе Р4Н3.

Наличие у НФ группы Р1Н1 ненулевых значений полярности и гидрофильности можно связать с присутствием следов детергентов, применявшихся при нанесении этих фаз на гидрофильный носитель.

Можно выделить группу полисилоксановых фаз с различными заместителями (№№ 2, 3, 4, 5, 6, 8, 9, 10). Их положение на карте селективности наглядно демонстрирует рост характеристик в зависимости от количества и полярности функциональных групп.

Группа одинаковых по составу фаз представлена в блоке А - НФ №№ 18, 19, 20, 21 и 23. Это полиэтиленгликолевые (ПЭГ) фазы, различающиеся молекулярной массой и достаточно близкие по характеристикам селективности. Эти фазы селективны к кислородсодержащим соединениям; по нашей классификации они все относятся к группе Р3Н2, т.е. обладают сильной полярностью и средней гидрофильностью.

Высокополярные и сильногидрофильные фазы группы Р4Н3 имеют сложную структуру с большим количеством нитрильных (№№ $15,16,17)$ или гидроксильных групп (№14).

Теоретическая оценка характеристик селективности НФ на примере ПЭГ. Карта селективности НФ может быть рассчитана теоретически, если известна структурная формула НФ, попытка такого расчета сделана в работе [26].

Приведем здесь расчет a priori характеристик полярности и гидрофильности НФ для ПЭГ-фаз, фрагментом структурной формулы которых является мономер -О$\mathrm{CH}_{2}-\mathrm{CH}_{2}$ - или диэтиловый эфир. Дипольный момент диэтилового эфира, взятый из справочных данных [34] равен 1.15 D. Обобщенный заряд рассчитывается по формулам $(4-7,31)$. Из этих данных по формуле (18) находим полярность 
$v_{0}=\frac{1.15^{2}}{7.19}=0.18$. Величина $n_{H}$ также рассчитывается из строения мономера по формуле (12) для одного атома кислорода, входящего в мономер. По формуле (16) считаем гидрофильность $w_{0}=\frac{n_{H}}{7.19}=\frac{0.233}{7.19}=0.032$. В сравнении с рассчитанными из индексов Ковача характеристиками ПЭГ фаз $\left(v_{0}=0.12 ; w_{0}=0.04\right)$ сделанная оценка довольно груба, однако может быть улучшена при более подробном рассмотрении структурных особенностей полимеров.

Сравнение нового метода трехпараметрической характеристики с методом Роршнайдера-МакРейнольдса. Для сравнения методов ТПХ и РоршнайдераМакРейнольдса (РМР) были выбраны 2 реперные молекулы метода РМР - 1-бутанол и 2-пентанон [35], которые относятся к двум разным множествам $\left(M_{0}, M_{1}\right)$ метода ТПХ. Величины полярности и гидрофильности в первом случае определялись по формулам таб.3 из индексов Ковача, а во втором случае - по формулам таб.4 из констант МакРейнольдса. В таблице 6 представлены характеристики полярности и гидрофильности НФ, обозначенные как «ТПХ»- рассчитанные из индексов Ковача, и как «РМР»- рассчитанные из констант МакРейнольдса.

Таблица 6. Характеристики селективности НФ, полученные методами ТПХ и РМР: $v_{0(T \Pi X)}$ и $w_{0(T \Pi X)}$ - значения, рассчитанные из индексов Ковача, $v_{0(P M P)}$ и $w_{0(P M P)}-$ из констант РМР.

\begin{tabular}{|c|c|c|c|c|}
\hline HФ & $v_{O(T I X)}$ & $w_{O(T \Pi X)}$ & $v_{O(P M P)}$ & $w_{O(P M P)}$ \\
\hline OV-7 & 0.034 & 0.015 & 0 & 0.008 \\
\hline DC-710 & 0.047 & 0.017 & 0.008 & 0.011 \\
\hline OV-25 & 0.067 & 0.019 & 0.028 & 0.017 \\
\hline XE-60 & 0.108 & 0.028 & 0.073 & 0.033 \\
\hline OV-225 & 0.107 & 0.021 & 0.072 & 0.016 \\
\hline Silar-5 CP & 0.139 & 0.033 & 0.106 & 0.033 \\
\hline Versilub F-50 & 0 & 0.014 & 0 & 0.002 \\
\hline Ucon 50HB-280X & 0.073 & 0.031 & 0.035 & 0.028 \\
\hline PEG 6000 & 0.117 & 0.041 & 0.082 & 0.039 \\
\hline PEG 4000 & 0.118 & 0.041 & 0.084 & 0.040 \\
\hline SILAR-7 CP & 0.182 & 0.042 & 0.152 & 0.041 \\
\hline Diglycerol & 0.170 & 0.055 & 0.139 & 0.057 \\
\hline Cyanoethylsucrose & 0.229 & 0.056 & 0.202 & 0.058 \\
\hline THEED & 0.187 & 0.061 & 0.186 & 0.061 \\
\hline PEG 20M & 0.116 & 0.040 & 0.082 & 0.039 \\
\hline
\end{tabular}

На рисунке 2 показано сравнение значений полярности НФ, рассчитанных двумя способами. Наблюдается сдвиг на постоянную величину (приблизительно 0.03). Такой сдвиг обусловлен тем, что в методе РМР за полярность отвечают несколько эталонов, и на 2-пентанон приходится только часть полярности в этой схеме. Это сравнение указывает на избыточность метода РМР в отношении характеристики полярности.

На рисунке 3 демонстрируется корреляция значений гидрофильности, рассчитанных по методу ТПХ и пересчитанных из констант РМР. В схеме РМР есть только один эталон, являющийся донором водородной связи (1-бутанол), и отсутствует пе- 
рераспределение характеристики гидрофильности между другими молекулами. Поэтому сравнение расчетов разными методами не дает сдвига. Небольшое расхождение значений в начале координат связано с постулированием в методе РМР сквалана как эталона неполярных фаз. В реальности неполярные фазы имеют примеси детергентов, придающих им небольшую гидрофильность.

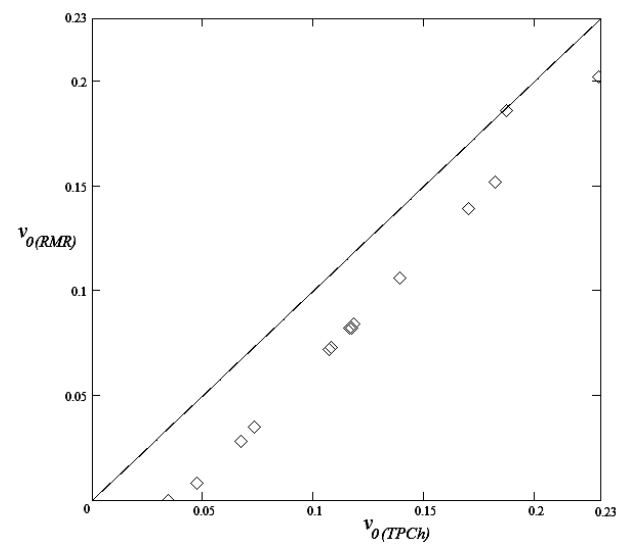

Рис. 2. Корреляция значений полярности: $v_{0(т п X)}$ - значения полярности $v_{0}$, рассчитанные из индексов Ковача, $v_{O(P M P)}$ - рассчитанные из констант РМР.

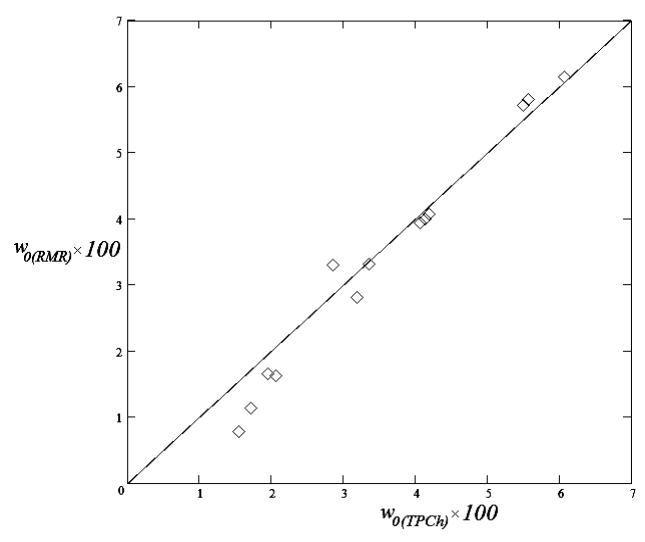

Рис. 3. Корреляция значений гидрофильности: $w_{0(т п X)}$ - значения гидрофильности $w_{0}$, рассчитанные из индексов Ковача, $w_{0(P M P)}$ - рассчитанные из констант РМР.

Таким образом, полученные корреляции указывают на согласованность методов ТПХ и РМР, избыточность метода РМР при характеристике полярности и возможность определения характеристик полярности и гидрофильности НФ из констант Роршнайдера-МакРейнольдса.

Использование карты селективности в выборе НФ. Для того, чтобы выбрать наиболее подходящую НФ для разделения заданной группы аналитов, необходимо рассчитать характеристики полярности и гидрофильности как для НФ, так и для молекул аналитов; характеристики молекул рассчитываются из их структурной формулы, способ расчета характеристик НФ не имеет значения. Стоит отметить, что молекулы аналита при расчете рассматриваются как конденсированная фаза, такая же как и НФ. На карту селективности наносятся характеристики НФ и молекул аналитов. Далее, следуя эмпирическому принципу «подобное в подобном», выбирается НФ, наиболее близко расположенная к группе целевых аналитов на карте селективности.

Для демонстрации способа выбора НФ с использованием метода ТПХ были выбраны 2 трудноразделяемые молекулы - пропилацетат и этилпропионат, являющиеся изомерами. Нет информации о разделении этих молекул из-за близких значений индексов Ковача. Они имеют высокую полярность, превышающую значения полярности, рассчитанные для фаз. Это значит, что необходимо выбрать НФ с максимальным значением полярности; также должны быть максимально близки значения гидрофильности у НФ и молекул аналитов. Из рисунка видно, что две фазы из присутствующих имеют максимально близкую полярность к разделяемой паре аналитов - TCEP и Cyanoethylsucrose, согласно таблице 5 у TCEP значение гидрофильности выше, а полярность ниже, чем у Cyanoethylsucrose. Из этого следует, что наилучшей НФ для разделения данных аналитов будет Cyanoethylsucrose, имеющая наиболее близкие значения характеристик полярности и гидрофильности к аналогичным характеристикам аналитов. 


\section{Заключение}

Описанный в статье метод трехпараметрической характеристики неподвижных фаз в газовой хроматографии основан на теории межмолекулярных взаимодействий, учитывающей неполярные, полярные силы и водородные связи. Для описания неполярных взаимодействий и введения теоретических характеристик применена теория обобщенных зарядов. На основании квантовомеханической оценки учитывается вклад в энергию ММВ водородных связей. Метод ТПХ является удобным при получении характеристик фаз из экспериментальных данных по индексам удерживания, способен дать теоретическую оценку обобщенного заряда, полярности и гидрофильности по молекулярному строению фазы и хорошо согласуется с соответствующими характеристиками метода Роршнайдера-МакРейнольдса. Метод ТПХ позволяет классифицировать НФ в удобном графическом виде - в виде карты селективности. Проведенная классификация фаз позволяет выбрать фазу, необходимую для определения трудноразделяемых аналитов по принципу «подобное в подобном».

\section{Работа выполнена при финансовой поддержке РФФИ (грант 18-03-00382a).}

\section{Список литературы}

1. Rohrschneider L. // J.Chomatogr. A. 1965. Vol. 17. pp. 1-12.

2. McReynolds W.O. // J.Chromatogr. A. 1970. Vol. 8. pp. 337-345.

3. Схунмакерс П., Оптимизация селективности в хроматографии // Пер. с англ. Под ред. В.А. Даванкова. М., Мир. 1989. 399 с.

4. Vitha M., Carr P.W. // J.Chromatogr. A. 2006. Vol. 1126. pp. 143-194.

5. Abraham M.H., Ibrahim A., Zissimos A.M. // J.Chromatogr. A. 2004. Vol. 1037. pp. 29-47.

6. Pierotti R.A. // Chem. Rev. 1976. Vol. 76. pp. 717-726.

7. Platts J.A., Abraham M.H., Butina D., Hersey A. // Journal of Chemical Information and Computer Sciences. 2000. Vol. 40. pp. 71-80.

8. Shevchik J., Lowventup M.S.H. // J.Chromatogr. A. 1981. Vol. 217. pp. 139-150.

9. Долгоносов А.М., Рудаков О.Б., Прудковский А.Г. Колоночная аналитическая хроматография: практика, теория, моделирование. СПб: Лань. 2015. 468 с. ISBN: 978-58114-1870-1.

10.Шатц В.Д., Сахартова О.В. Высокоэффективная жидкостная хроматография. Рига: Зинатне. 1988. 390 с.

11.Program for the calculation $C \log P$ download here. Available at: http://www.biobyte.com /index.html accessed 2 October 2019.

12.Platts J.A., Abraham M.H., Butina D., Hersey A. // Journal of Chemical Information and Computer Sciences. 2000. Vol. 40. pp. 71-80.
13. Abraham M.H., Chadha H.S., Leo A.J. // J.Chromatogr. A. 1994. Vol. 685. pp. 203-211.

14. Abraham M.H., Chadha H.S., Whiting G.S., Mitchell R.C. // J. Pharm. Sci. 1994. Vol. 83. pp. 1085-1100.

15.Lombardo F., Shalaeva M.Y., Tupper K.A., Gao F. et al. // J. Med. Chem. 2000. Vol. 43. pp. 2922-2928.

16.Dias N.C., Poole C.F. // J. Planar. Chromatogr. 2000. Vol. 13. pp. 337-347.

17. Долгоносов А.М., Зайцева Е.А. // Сорбиионные и хроматографические прочессы. 2014. T. 14. № 4. С. 578-590.

18.Долгоносов А.М., Зайцева Е.А. // Сорбиионные и хроматографические прочессы. 2015. T. 15. № 3. С. 321-332.

19. Долгоносов А.М., Зайцева Е.А. // Becmник Университета Дубна. 2015. № 1. С. 3641.

20. Долгоносов А.М. // Сорбиионные и хроматографические проиессы. 2015. Т. 15. № 3. C. 312-320.

21.Каплан И.Г. Межмолекулярные взаимодействия. Физическая интерпретация, компьютерные расчеты и модельные потенциалы. М.: БИНОМ. Лаборатория знаний. 2012. $394 \mathrm{c}$.

22.Reinganum M. // Ann.d.Physik. 1912. Vol.38. p. 649.

23.Keesom W.H. // Phys.Z. 1921. Vol.22. p.129.

24.Debye P. // Phys.Z. 1920. Vol.21. p.178. 
25.Falkenhagen M. // Phys.Z. 1922. Vol.23. p.87.

26.Зайцева Е.А., Долгоносов А.М. // Сорбиионные и хроматографические прочессы. 2018. T. 18. № 5. С. 676-689.

27.Долгоносов А.М. Модель электронного газа и теория обобщенных зарядов для описания межатомных взаимодействий и адсорбции. М. ЛИБРОКОМ. 2009. 167 с.

28.Долгоносов А.М. Неспецифическая селективность в проблеме моделирования высокоэффективной хроматографии. М., КРАСАНД. 2012. 256 c.

29.Dolgonosov A.M. // Protect. Metals \& Phys. Chem. Surfaces. 2015. No 51. pp. 951956. DOI: $10.1134 / \mathrm{S} 2070205115060052$.

30.Долгоносов А.М., Прудковский А.Г. // Российский журнал физической химии. 2006.

\section{References}

1. Rohrschneider L., J.Chomatogr. A, 1965 , Vol. 17, pp. 1-12.

2. McReynolds W.O., J.Chromatogr. A, 1970, Vol. 8, pp. 337-345.

3. Skhunmakers P., Optimizaciya selektivnosti v khromatografii, Per. s angl. Pod red. V.A. Davankova. M., Mir. 1989, 399 p.

4. Vitha M., Carr P.W., J.Chromatogr. A, 2006, Vol. 1126, pp. 143-194.

5. Abraham M.H., Ibrahim A., Zissimos A.M., J.Chromatogr. A, 2004, Vol. 1037, pp. 29-47.

6. Pierotti R.A., Chem. Rev., 1976, Vol. 76, pp. 717-726.

7. Platts J.A., Abraham M.H., Butina D., Hersey A., Journal of Chemical Information and Computer Sciences, 2000, Vol. 40, pp. 71-80.

8. Shevchik J., Lowventup M.S.H., J.Chromatogr. A, 1981, Vol. 217, pp. 139-150.

9. Dolgonosov A.M., Rudakov O.B., Prudkovskij A.G.,Kolonochnaya analiticheskaya khromatografiya: praktika, teoriya, modelirovanie, SPb, Lan', 2015, 468 p. ISBN: 978-5-81141870-1.

10. Schatz V.D., Sahartova O.V., Vysokoeffektivnaya zhidkostnaya khromatografiya, Riga, Zinatne, 1988, $390 \mathrm{p}$.

11. Program for the calculation ClogP download here. Available at: http://www.biobyte.com/index.html accessed 2 October 2019.

12. Platts J.A., Abraham M.H., Butina D., Hersey A., Journal of Chemical Information
Вып. 80. № 6. С. 964-968. ISSN 0036-0244. DOI: $10.1134 / \mathrm{S} 0036024406060227$.

31.Cambridge soft - chemical drawing, Chemical databases, Enterprises solutions, Desktop software, Consulting services. Available at: http://www.cambridgesoft.com accessed 2 October 2019.

32.Dolgonosov A.M. // Rus. Chem. Bul. 2016. No 65. pp 952-963.

33.McReynolds W.O. "Gas Chromatographic Retention Data". Preston. Technical Abstracts Co. Niles. 1966. 3th ed. 335 p.

34.Dean J.A. Lange's handbook of chemistry. Fifteenth Edition. 1992. McGRAW-HILL. INC. ISBN 0-07-016384-7.

35. Official website Shinwa Chemical Industries Ltd., RMR constants for SP. Available at: http://www.shinwa-cpc.co.jp/en/products/gc/ packed/stationary/ accessed 2 October 2019.

and Computer Sciences, 2000, Vol. 40, pp. 7180.

13. Abraham M.H., Chadha H.S., Leo A.J., J.Chromatogr. A, 1994, Vol. 685, pp. 203-211.

14. Abraham M.H., Chadha H.S., Whiting G.S., Mitchell R.C., J. Pharm. Sci.,1994, Vol. 83, pp. 1085-1100.

15. Lombardo F., Shalaeva M.Y., Tupper K.A., Gao F., Abraham M.H., J. Med. Chem., 2000, Vol. 43, pp. 2922-2928.

16. Dias N.C., Poole C.F., J. Planar. Chromatogr., 2000, Vol. 13, pp. 337-347.

17. Dolgonosov A.M., Zaitceva E.A., Sorbtsionnye i khromatograficheskie protsessy, 2014, Vol. 14, No 4, pp. 578-590.

18. Dolgonosov A.M., Zaitceva E.A., Sorbtsionnye i khromatograficheskie protsessy, 2015, Vol. 15, No 3, pp. 321-332.

19. Dolgonosov A.M., Zaitceva E.A., Vestnik Universiteta Dubna, 2015, No 1, pp. 36-41.

20. Dolgonosov A.M., Sorbtsionnye i khromatograficheskie protsessy, 2015, Vol. 15, No 3, pp. 312-320.

21. Kaplan I.G. Mezhmolekulyarnye vzaimodejstviya. Fizicheskaya interpretaciya, komp'yuternye raschety i model'nye potencialy / I.G. Kaplan ; per. S angl., M., BINOM, Laboratoriya znanij, 2012, $394 \mathrm{p}$.

22. Reinganum M., Ann.d.Physik, 1912, Vol. 38, p. 649.

23. Keesom W.H., Phys.Z., 1921, Vol. 22, p. 129.

24. Debye P., Phys.Z, 1920, Vol.21, p. 178. 
25. Falkenhagen M., Phys.Z, 1922. Vol.23. p.87.

26. Zaitceva E.A., Dolgonosov A.M., Sorbtsionnye i khromatograficheskie protsessy, 2018, Vol. 18, No 5, pp. 676-689.

27. Dolgonosov A.M., Model' elektronnogo gaza i teoriya obobshchennyh zaryadov dlya opisaniya mezhatomnyh vzaimodejstvij i adsorbcii, M., LIBROKOM, 2009, pp. 167.

28. Dolgonosov A.M., Nespecificheskaya selektivnost' $\mathrm{v}$ probleme modelirovaniya vysokoeffektivnoj khromatografii. M., KRASAND, 2012. $256 \mathrm{p}$.

29. Dolgonosov A.M., Protect. Metals \& Phys. Chem. Surfaces, 2015, Vol. 51, pp. 951956. DOI: $10.1134 /$ S2070205115060052.

30. Dolgonosov A.M., Prudkvskij A.G., Rossijskij zhurnal fizicheskoj khimii, 2006, Vol. 80, No 6, pp. 964-968. ISSN 0036-0244. DOI: $10.1134 /$ S0036024406060227.

Зайцева Елена Александровна - аспирантка четвертого года обучения, лаборатория сорбционных методов, Институт геохимии и аналитической химии им. В.И. Вернадского РАН (ГЕОХИ РАН), Москва

Долгоносов Анатолий Михайлович - д.х.н., ведущий научный сотрудник, лаборатория сорбционных методов, Институт геохимии и аналитической химии им. В.И. Вернадского РАН (ГЕОХИ РАН), Москва.
31. Cambridge soft - chemical drawing, Chemical databases, Enterprises solutions, Desktop software, Consulting services. Available at: http://www.cambridgesoft.com accessed 2 October 2019.

32. Dolgonosov A.M.,Rus. Chem. Bul., 2016, Vol. 65, pp. 952-963.

33. McReynolds W.O. "Gas Chromatographic Retention Data", Preston. Technical Abstracts Co., Niles, 1966, 3th ed. 335.

34. Dean J. A. LANGE'S HANDBOOK OF CHEMISTRY, Fifteenth Edition (1992), McGRAW-HILL, INC. ISBN 0-07-016384-7.

35. Official website Shinwa Chemical Industries Ltd., RMR constants for SP. Available at: http://www.shinwa-

cpc.co.jp/en/products/gc/packed/stationary/ accessed 2 October 2019.

Zaitceva Elena A. - PhD student, Lab of Sorption Methods, Vernadsky Institute of Geochemistry and Analytical Chemistry Russian Academy of Sciences (GEOKHI RAS), Moscow, lil-dante@mail.ru

Dolgonosov Anatoly M. - Dr.Sci.(Chem.), Leading scientific researcher, Lab of Sorption Methods, Vernadsky Institute of Geochemistry and Analytical Chemistry Russian Academy of Sciences (GEOKHI RAS), Moscow, amdolgo@mail.ru 